Revue d'histoire de l'Amérique française

REVUE D.HISTOIRE DE L'AMÉRIQUE FRANÇAISE

\title{
La lutte pour le droit à l'avortement (1969-1981)
}

\section{Diane Lamoureux}

Volume 37, numéro 1, juin 1983

URI : https://id.erudit.org/iderudit/304126ar

DOI : https://doi.org/10.7202/304126ar

Aller au sommaire du numéro

Éditeur(s)

Institut d'histoire de l'Amérique française

ISSN

0035-2357 (imprimé)

1492-1383 (numérique)

Découvrir la revue

Citer cet article

Lamoureux, D. (1983). La lutte pour le droit à l'avortement (1969-1981). Revue d'histoire de l'Amérique française, 37(1), 81-90. https://doi.org/10.7202/304126ar d'utilisation que vous pouvez consulter en ligne.

https://apropos.erudit.org/fr/usagers/politique-dutilisation/ 


\section{LA LUTTE POUR LE DROIT À L'AVORTEMENT $(1969-1981)^{*}$}

DIANE LAMOUREUX

La lutte pour le droit à l'avortement libre et gratuit a constitué une des principales manifestations publiques de l'existence d'un mouvement féministe au Québec. D'un côté, depuis 1969, la question de l'avortement a toujours été présente dans les préoccupations des courants les plus «radicaux» du mouvement. De l'autre, c'est en grande partie à travers la structuration d'un mouvement national pour la liberté d'avortement que les courants radicaux du féminisme ont pu se consolider en dehors de la région métropolitaine. Deux phénomènes méritent d'être examinés.

Le premier concerne la lutte elle-même. On pourrait y distinguer cinq phases: un service de référence dans l'attente de modifications législatives; une action défensive lorsque le fonctionnement du service de référence est entravé par la répression étatique; une mobilisation limitée; une extension des alliances; finalement, la pratique d'avortements par des militants féministes. Cette évolution est illustrée à travers l'expérience du mouvement montréalais.

Le deuxième phénomène, c'est l'effet produit sur le mouvement par la persistance d'une telle constante de réflexion. À cet égard, deux éléments semblent devoir retenir l'attention. D'une part, des changements dans la formulation de la problématique concernant l'avortement, qu'on peut rattacher aux mutations d'aide idéologique intervenues au sein du mouvement féministe québécois. D'autre part, le rôle de leadership informel joué par le comité de lutte pour l'avortement libre et gratuit à l'intérieur d'un mouvement féministe particulièrement morcelé.

Avant d'entreprendre cette démonstration, il serait cependant utile de préciser ce qu'on entend par mouvement féministe. On pourra ainsi éviter les quiproquos et les fausses polémiques. Le féminisme, c'est le mouvement de lutte contre l'organisation patriarcale des sociétés contemporaines. Ceci implique deux postulats de base: toute transformation sociale véritable doit passer par l'élimination de l'antagonisme

\footnotetext{
* Communication présentée dans le cadre du colloque de l'Institut d'histoire de l'Amérique française, Montréal, 22-23 octobre 1982.
} 
hommes/femmes; l'autonomie politique et organisationnelle des femmes est essentielle pour mener à terme un tel type de transformation. Ce projet est porté par ce qu'il est désormais courant d'appeler les «groupes autonomes de femmes».

\section{Les zigzags de parcours}

Si l'importance du phénomène de l'avortement n'a pas à être démontrée, il n'en reste pas moins que ce n'est qu'au début des années '70 que ce problème "privé» devient l'enjeu d'un débat public. En 1969, un étudiant et une étudiante de McGill, sous l'impact des luttes qui se meuvent alors aux États-Unis, publient une brochure sur la contraception et l'avortement. Cette brochure est rapidement traduite en français par les membres du Front de libération des femmes, qui très vite prennent conscience de l'ampleur du phénomène. À peine la brochure commence-t-elle à circuler que le FLF reçoit des appels téléphoniques de femmes s'informant des possibilités d'obtenir un avortement.

C'est là qu'est né le service de référence pour avortement assumé tour à tour par le Front de libération des femmes, le Centre des femmes et finalement le Comité de lutte pour l'avortement libre et gratuit. Grosso modo, la référence comprend deux volets. Le premier est d'offrir un service effectif aux femmes qui en font la demande. Dans ce sens, le service de référence s'apparente à un secrétariat médical, où on évalue plus concrètement les besoins précis des femmes concernées et dirige celles-ci vers les ressources appropriées: médecins pratiquant l'avortement dans des cliniques privées, hôpitaux anglophones, New York. L'autre volet de la référence, plus politique celui-là, consiste à faire en sorte que des femmes qui font face à une situation commune se rencontrent et puissent discuter de leur problème à l'occasion d'une séance d'information sur l'avortement comme phénomène médical.

Comment expliquer qu'au Québec on se soit cantonné dans la référence, alors qu'ailleurs (France et Italie, par exemple) les femmes qui revendiquaient la liberté d'avortement ont également entrepris de pratiquer des avortements. Ce geste peut s'expliquer par le contexte. En 1968, au nombre des recommandations de la Commission Bird (Commission royale d'enquête sur la situation de la femme au Canada) figure la décriminalisation de l'avortement. En 1969, l'État de New York légalise la pratique d'avortements dans les hôpitaux. Le FLF estime que l'avortement est susceptible d'être légalisé dans un avenir rapproché au Québec et qu'il vaut donc mieux concentrer les efforts de mobilisation ailleurs.

Certes, le FLF va tenter sporadiquement de «mobiliser» autour de cette question. La cellule «avortement» s'occupera donc officiellement et du volet référence et du volet mobilisation. Mais les militantes plus 
intéressées à la mobilisation quitteront rapidement le FLF après avoir critiqué son mode de fonctionnement ${ }^{1}$, ce qui ne laissera subsister que le volet référence qui, entre autres, permet au FLF de maintenir pignon sur rue.

Durant cette période, le FLF n'est toutefois pas la seule organisation qui prend des positions pro-avortement. Des intervenantes et intervenants dans les services de santé et des militantes trotskystes forment un groupe de pression, le Front commun pour l'abrogation des lois sur l'avortement (FCALA), qui tente de porter le débat devant l'opinion publique. Il organise quelques conférences de presse ou manifestations réunissant à peine une centaine de personnes. L'essentiel de l'action effective du FCALA se résume donc à une politique de lobbying auprès du gouvernement fédéral.

La volonté de mobilisation, suscitée en grande partie par une intervention extérieure au mouvement des femmes, le force à réagir. Tant que la référence était tolérée ou niée par la conspiration du silence des autorités, il était même préférable de ne pas trop mobiliser afin de ne pas mettre en péril cette brèche fragile instaurée par la femme dans le contrôle social sur son corps. C'est la fin de la tolérance étatique qui a forcé le service de référence à réagir et à créer des conditions de mobilisation suffisantes pour permettre la poursuite de ses activités.

Ce processus est très bien décrit dans un dossier du Comité de lutte pour l'avortement libre et gratuit ${ }^{2}$. On y souligne le rôle joué par l'arrestation du docteur Morgentaler et le début des procès contre d'autres médecins pratiquant des avortements dans leurs bureaux, et surtout par la perquisition au Centre des femmes, qui assure à cette époque le service de référence. «C'est sans doute à ce moment que les militantes ont vraiment été saisies de l'illégalité et de la clandestinité du service. Perquisitionnées mais isolées, sur qui pouvaient-elles compter? Comment réagir?» ${ }^{3}$

L'initiative d'entreprendre la mobilisation ne vient cependant pas du Centre, pris de court par la disparition de ses médecins-ressources et forcé par la logique du service à trouver des solutions de remplacement pour continuer à pratiquer des avortements. C'est au FCALA que revient l'initiative de convoquer, à l'automne 1973, une assemblée publique afin de former le Comité de défense de Morgentaler (CDF). Ce comité se fixe pour objectif de rassembler ses forces pro-avortement et réunit au départ, outre le FCALA et le Centre des femmes, des militantes de la CEQ, du ADDS ainsi que des personnes sans affiliation organisationnelle.

\footnotetext{
Voir à ce sujet Véronique O’Leary et Louise Toupin, Québécoises deboutte!, 1: 94-95. Dossier spécial sur l'avortement et la contraception libres et gratuits, 8 mars 1975. Idem, 41 .
} 
Les divergences n'ont cependant pas tardé à faire surface. Le CDF reproche aux membres du FCALA, qui contrôlent le Comité de défense de Morgentaler, de se limiter à la défense des médecins arrêtés et de ne pas inscrire au nombre de ses revendications la contraception et l'avortement libres et gratuits. Plus particulièrement, les militantes du Centre ont l'impression que l'enjeu de la lutte n'est pas défini clairement. «Nous ne voulons pas concentrer la lutte uniquement autour de la défense des médecins. Il ne s'agit pas de défendre le droit des médecins à pratiquer des avortements, mais bien de s'assurer à nous les femmes, le droit d'avorter librement et gratuitement. ${ }^{4}$

Les militantes du Centre, conjointement avec des membres du comité Laure-Gaudreault de la CEQ et certaines femmes des ADDS, décident donc de former leur propre comité de mobilisation, le Comité de lutte pour l'avortement libre et gratuit qui voit le jour au printemps 1974.

Dans un premier temps, le Comité de lutte veut cerner de plus près les enjeux de la lutte pour l'avortement, préalable essentiel selon lui, à toute mobilisation sur la question. Ce travail de déblayage théorique, de même que les démêlés avec la gauche de l'époque mobilisent en grande partie l'énergie du Comité au cours de la période 1974-1976. Le travail de sensibilisation en dehors de cercles militants est assumé essentiellement par le Théâtre des cuisines, qui entreprend une tournée avec sa pièce d'agit-prop: «Nous aurons les enfants que nous voulons.»

Ce n'est qu'avec la disparition des aspects les plus criants de la répression, après l'arrivée au pouvoir du Parti québécois, et l'arrêt des poursuites contre Morgentaler que le Comité de lutte juge la situation suffisamment favorable pour lancer une campagne de mobilisation sur la question de l'avortement. Cette dernière prend d'abord la forme d'une pression accrue sur le Parti québécois. Dans le passé, l'on insistait sur le fait que c'était une loi fédérale qui limitait sérieusement les possibilités légales d'avortement au Québec. On disait aussi que la situation était aggravée au Québec par le poids de la tradition catholique. À partir de l'élection du Parti québécois en novembre 1976, on met plutôt l'accent sur les contradictions internes de ce «non gouvernement» d'un parti indépendantiste dans le contexte d'un État fédéral.

$\mathrm{Au}$ lieu de s'effectuer sur le gouvernement fédéral, les pressions portent désormais essentiellement sur le gouvernement provincial. Le premier niveau d'intervention est donc celui des conflits de juridiction entre le gouvernement provincial et le gouvernement fédéral. La loi sur l'avortement relève du code criminel et est donc de juridiction fédérale. Les poursuites contre les contrevenants à la loi relèvent du procureur général de chaque province. De même, le statut d'hôpital est donné à

4 Cité dans Dossier avortement-référence, janvier 1978, 2. 
un établissement de santé en vertu de la juridiction provinciale. Dans ces circonstances, la tactique qui est mise de l'avant par le mouvement est de faire pression sur le gouvernement québécois. Il lui demande d'abroger, dans les faits, la loi fédérale en ce qui concerne le Québec et de montrer à quel point les Québécois sont systématiquement défavorisés par l'application de cette loi.

Ce type d'intervention permet au mouvement pour le droit à l'avortement de se situer au coeur du débat sur la question nationale, et ce, par deux biais. Premièrement, le mouvement joue sur les conflits de pouvoir. À ce niveau, on est loin du débat de 1970 autour de la caravane pour l'avortement, à laquelle le FLF a refusé de participer, alléguant ne pas reconnaître le gouvernement fédéral comme gouvernement légitime du peuple québécois ${ }^{5}$. Cette initiative évite la «marginalisation» par rapport au débat sur la question nationale tout en permettant d'intégrer à la mobilisation pour le droit à l'avortement plusieurs militantes péquistes. Deuxièmement, le mouvement pour le droit à l'avortement conserve ainsi la possibilité de s'insérer dans le débat non moins important de l'après-indépendance.

Soulignons enfin le fait que ce type d'intervention permet aux forces pro-avortement à l'intérieur du Parti québécois de forcer, malgré la direction, une prise de position en faveur de l'avortement libre et gratuit. Sans surestimer l'impact d'une telle prise de position, on peut tout de même penser qu'elle contribue à susciter un éventuel déblocage sur la question, en même temps que la vaste opposition rencontrée fonde tout le discours sur la nécessité d'une mobilisation.

C'est en continuité avec cette nouvelle analyse qu'est mise sur pied, au printemps 1978, la Coordination nationale pour l'avortement libre et gratuit. Le geste représente une rupture à deux niveaux. D'une part, en mettant sur pied la Coordination, une certaine frange du mouvement féministe fait de la lutte pour l'avortement sa priorité de mobilisation. D'autre part, on essaie d'engager dans la lutte d'autres secteurs que le mouvement des femmes, puisque la Coordination regroupe également des représentantes des centrales syndicales, des intervenantes dans les CLSC, des organismes de planning familial et même des militantes des comités de condition féminine du Parti québécois.

La constitution de la Coordination va permettre au mouvement de faire des pas importants. D'abord, on peut constater par ce biais la capacité de mobilisation du mouvement et ce, à des niveaux très différents: au niveau de la masse, avec les grandes manifestations de 1978 et surtout 1979; au niveau de l'impact public, avec la publication de la liste de 100 femmes connues affirmant avoir déjà eu recours à l'avortement et se prononçant en faveur de la liberté d'avortement. Mais plus

5 Voir Véronique O'Leary et Louise Toupin, op. cit., 71. 
profondément, la Coordination permet de mesurer la réalité véritablement nationale d'un mouvement féministe qui perd son caractère montréalais pour tenir un même langage aux quatre coins du Québec, comme le mettent en lumière la semaine nationale d'action de 1978 et la formation de regroupements régionaux de la Coordination.

Mais à un autre niveau, la Coordination rend également possible, en posant publiquement des actions sur la question de l'avortement, le développement de pratiques alternatives, et la mise sur pied de services d'avortement contrôlés par les femmes. On en est maintenant à cette cinquième étape de la lutte. Cette prise en charge de la question de l'avortement par les femmes représente nettement un pas en avant par rapport au début des années '70, quoique rien ne soit définitivement acquis à cet égard. Les centres de santé de femmes fonctionnent avec la tolérance du pouvoir et il se pourrait bien que cette tolérance n'ait qu'un temps, d'autant plus que la mobilisation tend à s'estomper sur cette question.

\section{Les discours féministes}

Reste maintenant à savoir le contenu que le mouvement féministe donne à cette lutte pour le droit à l'avortement. On a vu précédemment que ce n'est qu'avec la formation de la Coordination pour l'avortement libre et gratuit que le discours féministe sur l'avortement acquiert une certaine cohérence, quoique certains discours du FLF sur la famille puissent apparaître, avec le recul des années, plus avant-gardistes que bien des discours contemporains sur la maternité librement consentie. Il n'en demeure pas moins que le discours féministe s'articule fondamentalement autour de deux pôles: la maternité librement choisie et l'autonomie des femmes, ce qui donne naissance aux deux principaux slogans du mouvement: «Nous aurons les enfants que nous voulons» et «La politique sur notre ventre ne se fera pas sur notre dos».

Le thème de la maternité librement consentie est celui sur lequel l'insistance est mise tout au cours des mobilisations pour le droit à l'avortement. Il est nécessaire de s'y attarder également parce qu'il permet d'opérer la jonction avec d'autres problématiques qui ne sont pas uniquement propres aux femmes.

Le thème de la maternité librement consentie met l'accent sur une approche globale de la maternité, qui la situe dans son contexte économique et social. Revendiquer la maternité librement consentie, c'est affirmer que les femmes ne sont pas des machines à fabriquer des enfants et qu'elles ont une sexualité qui doit être séparée de la procréation. «Ce que nous voulons, c'est contrôler notre corps, décider de nos maternités et jouir de notre sexualité sans l'enfermer dans la production d'enfants», souligne le Comité de lutte. 
L'avortement n'apparaît donc pas comme une revendication isolée. Il permet plutôt d'insérer la lutte des femmes dans deux courants d'opinion plus larges. D'une part, dans un courant de plus grande tolérance en ce qui a trait à la sexualité, d'autre part, dans le mouvement pour le planning familial. Cette volonté de resituer la lutte des femmes dans un contexte plus global est d'ailleurs présente dans l'argumentation du dossier spécial publié en 1975. On peut y lire:

C'est pourquoi nous revendiquons: la création de centres de planification des naissances à travers tout le Québec, des cours sur la sexualité et la contraception obligatoires dans toutes les écoles, et ce, avant la puberté, et la gratuité des contraceptifs. ${ }^{6}$

Dans une telle perspective, la question de l'avortement est liée de très près à celle de la contraception. La très forte demande d'avortements qui parvient au Comité de lutte est perçue comme une conséquence inévitable de la faible diffusion de méthodes contraceptives à la fois efficaces et non dommageables. Ainsi, à travers la revendication du droit à l'avortement, est-il possible de dénoncer l'hypocrisie et l'irresponsabilité d'une société qui refuse à la fois l'éducation sexuelle et l'avortement.

Relier les questions de l'avortement et de la contraception amène le Comité de lutte à collaborer avec d'autres forces sociales, comme le mouvement pour le planning des naissances et certains CLSC qui publient un manifeste pour le planning des naissances. De plus, lors des séances d'information qui précèdent les avortements, on explique aux femmes non seulement ce qui se passera au moment de l'avortement, mais on les renseigne aussi sur les méthodes contraceptives.

Avec le développement des centres de santé de femmes et surtout des centres d'auto-santé, il s'effectue une remise en question de la contraception. Dans un texte qui a largement influencé les réflexions du Comité de lutte, un groupe de femmes italiennes dénonce la pénurie de moyens contraceptifs:

Nous aussi, comme toutes les femmes, nous trouvons dans la nécessité - urgente entre toutes — d'organiser la lutte pour l'avortement, étant donné que le niveau de la recherche médicale ne nous permet pas de mettre simplement en avant une diffusion libre et gratuite des moyens contraceptifs. En même temps, nous dénonçons le fait que jusqu'à présent, l'illégalité de l'avortement a joué un rôle de pilier central d'une entreprise de boucherie humaine, ceci dans la mesure où elle a servi à retarder et à décourager complètement la recherche de méthodes contraceptives qui ne détruisent pas la santé psychophysiologique des femmes. ${ }^{7}$

\footnotetext{
Dossier spécial..., op. cit., 23.

Lutte féministe, Maternité et avortement. Texte photocopié, Archives du Comité de lutte pour l'avortement libre et gratuit.
} 
Les coups les plus durs sont portés principalement contre la médecine officielle et l'Église catholique. De la loi comme telle, on parle de moins en moins, si ce n'est pour expliquer qu'elle est appliquée de façon particulièrement restrictive au Québec. Même ceci sert plus à désigner une cible de choix dans la morale catholique qu'à dénoncer la loi comme telle. L'insistance est de plus en plus mise sur le fait que l'influence du clergé est tellement persistante que seuls les hôpitaux anglophones de Montréal pratiquent réellement des avortements au Québec. D'autre part, les médecins sont très souvent dénoncés pour leur hypocrisie, puisqu'on insiste sur le fait que plusieurs médecins qui refusent d'avorter les femmes dans les hôpitaux, sous prétexte de considérations morales, sont pourtant bien disposés à le faire clandestinement, dans leur cabinet, moyennant rémunération.

Mais il faut également prendre en considération le discours sur l'autonomie des femmes. Ce discours est largement occulté par le premier puisque le Comité de lutte est la seule organisation à se battre pour l'obtention du droit à l'avortement. Avec la formation de la coordination nationale, on assiste à la prise en charge de la lutte par un groupe plus large, ce qui permet au comité de s'interroger sur les objectifs qu'il poursuit à travers cette lutte pour le droit à l'avortement. On assiste donc à une réapparition du discours sur l'autonomie des femmes, ce qui s'avère très important pour la poursuite de la mobilisation, suite au début de fonctionnement des cliniques Lazure. Elle revêt une importance déterminante dans la réorientation de la lutte vers la création d'un réseau de cliniques d'avortement contrôlé par les femmes.

Ainsi au comité, nous avons toujours tenté de définir notre lutte en partant de l'hypothèse que la reproduction biologique, c'est-à-dire la maternité, en tant que phénomène social, se retrouve à la racine de notre oppression et de notre exploitation, et ce à partir du moment où une classe dominante cherche à s'approprier et à contrôler la force de travail que nous produisons, et pour cela nous maintenir en esclavage (...) Le droit à l'avortement est une première étape vers la réappropriation de notre fonction de reproduction et vers notre libération. ${ }^{8}$

Cette deuxième composante du discours féministe rend beaucoup plus difficile une traduction institutionnelle des revendications des femmes puisqu'elle remet en cause les fondements mêmes du système patriarcal. C'est ce qui permet d'expliquer que ce soit là la composante la moins connue et la moins reprise du discours féministe sur l'avortement. Paradoxalement, on observe le phénomène suivant: pour gagner le droit à l'avortement, le mouvement féministe veut s'associer à d'autres forces sociales, mais en même temps, son discours de fond sur l'autonomie des femmes ne peut être repris en charge par ces autres forces

\footnotetext{
${ }^{8}$ Dossier avortement-référence, 2-3.
} 
sociales. Tout le débat public autour de la question de l'avortement tend donc à se résumer à la question de la maternité librement consentie, ce qui a pour effet d'occulter l'impact profond que pourrait avoir cette lutte dans le développement d'une conscience féministe de masse.

La formation de la Coordination nationale, en tant que processus d'association entre le mouvement féministe et d'autres composantes sociales, marque à la fois un pas en avant en ce qui concerne la mobilisation et un certain recul en ce qui a trait au discours, d'autant plus que la composante féministe de la Coordination doit effectuer des concessions quant aux objectifs de la lutte.

Cela apparaît clairement lors de la réunion de formation de la Coordination. Alors que le débat se polarise sur la question d'un réseau qui devrait être contrôlé, pour les unes, par les usagères et les femmes qui y travaillent, et pour les autres, par les usagères et le personnel. Loin d'être un simple débat sémantique, ceci témoigne de toute une vision de la lutte. Faut-il lutter pour l'autonomie des femmes, en mettant sur pied un réseau alternatif au réseau médical instauré par les cliniques Lazure, c'est-à-dire «démédicaliser» la question de l'avortement pour rendre aux femmes un certain contrôle sur leur corps; faut-il plutôt se fixer comme objectifs la seule obtention du droit à l'avortement, en laissant de côté, à tout le moins dans un premier temps, la critique des institutions médicales?

En optant pour la seconde position, la Coordination fait peut-être preuve d'un certain réalisme sur ce qui pourrait effectivement être obtenu, mais elle renonce du même coup à mettre de l'avant une perspective réellement radicale en ce qui concerne l'avortement. Cette évolution au sein de la Coordination accélère, par ailleurs, dans l'ensemble du mouvement féministe, la réflexion sur la nécessité, en même temps que se construit la mobilisation large autour d'un objectif précis, de poser les jalons d'une pratique autre, si l'on veut éviter la récupération.

Dans ce sens, le fait que la Coordination, comme organisme aux objectifs très larges, lutte pour un réseau public de cliniques d'avortement, alors que le Comité de lutte s'oriente d'abord vers la critique des cliniques Lazure, puis se dissout au profit de pratiques alternatives d'avortement, reflète un cheminement politique relativement important. Au lieu de chercher à concilier les deux pôles de la maternité librement consentie et du contrôle du corps, on essaie d'articuler deux pratiques différenciées.

\section{Un mouvement à but unique}

À la fin d'une telle analyse, on peut se pencher sur le rôle joué par le Comité de lutte par rapport à la composante radicale du mouvement féministe. Ce rôle peut être évalué à deux niveaux. D'une part, comme 
la question de l'avortement représente l'unique point de rencontre entre le mouvement féministe et d'autres forces sociales (principalement le mouvement syndical), le Comité de lutte apparaît souvent comme le porte-parole autorisé du féminisme auprès du mouvement syndical ou encore des organismes gouvernementaux. Ainsi, un rôle de direction ou, à tout le moins, de représentativité/crédibilité dans un contexte de morcellement du féminisme en plusieurs collectifs lui est conféré de l'extérieur.

D'autre part, cette reconnaissance extérieure repose sur certains éléments qu'on peut relier à la dynamique interne du mouvement féministe. Alors que, jusqu'en 1979, ce mouvement essaie de se constituer à partir d'un pôle central - le Front de libération des femmes ou le Centre des femmes - on peut noter en même temps le fonctionnement du Centre des femmes en plusieurs collectifs et l'apparition d'une série de collectifs à but unique, menant une intervention chacun de son côté. Dans ce centre, le Comité de lutte, comme principal héritier militant du Centre des femmes, représente à plusieurs égards une continuité organisationnelle avec la «tradition» féministe du début des années '70. Par ailleurs, et c'est là un élément encore plus central, le Comité de lutte, en mettant sur pied la Coordination, joue un rôle important dans le regroupement des forces féministes à Montréal. Ainsi, sans que la question ne soit véritablement posée, le Comité de lutte joue un rôle de leadership informel, en provoquant un regroupement sur ses propres bases.

Par ce biais, on peut examiner un autre point qui semble central lorsqu'on considère la lutte pour le droit à l'avortement. À bien des égards, la dynamique autour de la question de l'avortement s'apparente à celle qui caractérise le mouvement pour le droit de vote au début du siècle. Plus précisément, ces revendications qui, au départ, constituent une revendication parmi les autres, ont tendance à éclipser toutes les autres pour devenir, en quelque sorte, la seule image publique d'un mouvement au départ beaucoup plus diversifié. Cette dynamique semble cependant centrée ces dernières années, avec la formation de regroupements contre la violence faite aux femmes, mais il n'en reste pas moins que l'obtention du droit à l'avortement a, dans plusieurs pays, coïncidé avec une crise de perspective ou à tout le moins un essoufflement des mouvements féministes. 